\title{
Implantation of GL261 neurospheres into C57/BL6 mice: A more reliable syngeneic graft model for research on glioma-initiating cells
}

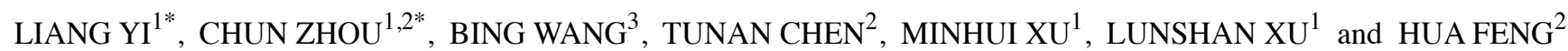 \\ ${ }^{1}$ Department of Neurosurgery, Daping Hospital, ${ }^{2}$ Department of Neurosurgery, \\ Southwest Hospital, Third Military Medical University; ${ }^{3}$ Department of Neurosurgery, \\ Yongchuan District People's Hospital, Chongqing, P.R. China
}

Received February 18, 2013; Accepted April 5, 2013

DOI: $10.3892 /$ ijo.2013.1962

\begin{abstract}
Recent studies have demonstrated that inflammatory cells and inflammatory mediators are indispensable components of the tumor-initiating cell (TIC) niche and regulate the malignant behavior of TICs. However, conventional animal models for glioma-initiating cell (GIC) studies are based on the implantation of GICs from human glioblastoma (GBM) into immunodeficient mice without the regulation of immune system. Whether animal models can mimic the cellular microenvironment of malignancy and evaluate the biological features of GICs accurately is unclear. Here, we detected the biological features of neurosphere-like tumor cells derived from the murine GBM cell line GL261 (GL261-NS) and from primary human GBM (PGBM-NS) in vitro, injected GL261-NS into syngeneic C57/BL6 mouse brain and injected PGBM-NS into NOD/SCID mouse brain, respectively. The tumorigenic characteristics of the two different orthotopic transplantation models were analyzed and the histological discrepancy between grafts and human primary GBM was compared. We found that GICs enriched in GL261-NS, GL261-NS and PGBM-NS exhibited increased GIC potential and enhanced chemoresistance in vitro. GL261-NS was significantly more aggressive compared to GL261 adhesive cells (GL261-AC) in vivo and the enhanced aggression was more significant in syngeneic mice compared to immunodeficient mice. The discrepancy of tumorigenicity between GL261-NS and GL261-AC in C57/BL6 mice was also larger compared to that between PGBM-NS and PGBM-AC in immunodeficient mice. Syngrafts derived from GL261-NS in C57/BL6 mice corre-
\end{abstract}

Correspondence to: Dr Hua Feng, Department of Neurosurgery, Southwest Hospital, Third Military Medical University, 30 Gaotanyan Main Street, Shapingba District, Chongqing 400038, P.R. China E-mail: happyape@126.com

*Contributed equally

Key words: cancer stem cell, glioma, GL261, inflammation sponded to the human GBM histologically better, compared with xenografts derived from PGBM-NS in NOD/SCID mice, which lack inflammatory cells and inflammatory mediators. We conclude that the inflammatory niche is involved in the tumorigenicity of GICs and implantation of GL261-NS into C57/BL6 mice is a more reliable syngeneic graft model for in vivo study on GICs relative to the immunodeficiency model.

\section{Introduction}

Although there has been progress in treatment in the recent decade, patients with glioblastoma (GBM) still only have a median survival of 9 months and 5-year survival rate of $9.8 \%$ (1). Exploration of glioma-initiating cells (GICs) impelled us to recognize the biological behavior of glioma from a new perspective. GICs are identified to be the root of tumorigenesis, invasion, angiogenesis and treatment resistance (2). It is also regarded as the important target to treat malignant glioma (3). Further investigation of GICs in vitro and in vivo may promote the effective strategies and methods to treat glioma.

The correct GICs sources and the suitable experimental animal models are necessary for research on GICs in vivo (4). It has been reported that GICs could be isolated from human primary glioma samples and glioma cell lines (5-7). A number of implanted models based on the injection of GICs into immunodeficient or immunocompetent mice are being used to investigate GICs in vivo (7-9). However, whether these implanted models can reflect malignant biological behavior of GICs accurately is unclear.

Although human primary glioma cells preserve the majority of glioma characteristics, the heterogeneous mesenchymal microenvironment and the deficiency of immunological elements may induce the large discrepancy of biological features between human primary glioma and xenograft in immunodeficient animal. Murine malignant glioma cell line GL261 was created by intracranial injection of 3-methylcholantrene into C57/BL6 mice and widely used in glioma research (10). In order to confirm a reliable experimental animal model for research on GICs, we isolated neurosphere-like tumor cells from GL261 (GL261-NS) and primary human glioma specimens (PGBM-NS), injected GL261-NS into C57/BL6 mice brain to 
establish a syngeneic orthotopically transplanted model and injected PGBM-NS into NOD/SCID mouse brain to establish a xenograft model. We detected the different tumorigenic characteristics of GICs and analyzed the tumor inflammatory microenvironment in the two models.

\section{Materials and methods}

Patients and specimens. Tumor tissues were obtained from the Neurosurgery Department of Daping Hospital. Five patients with primary GBM underwent curative resection between 2008 and 2009 and samples from these patients were used for primary glioma cell culture (Table I). The protocol was approved by the institutional Research Ethics Board at Daping Hospital, Chongqing, China.

Cell cultures. GL261 cell line was obtained from American Type Culture Collection (ATCC, Manassas, VA, USA). GL261-NS were obtained and cultured as we previously described (11). We cultured GL261 cells and decreased one half of FBS concentration every 3 days. GL261 cells were seeded into defined stem cell medium after 6 days. GICs from human glioma tissues and adhesive glioma cells were isolated and identified as we previously described (12). The primary tumor cells were detached with trypsin (Gibco) and suspended in defined stem cell medium described previously at a density of $1,000 \mathrm{cells} / \mathrm{ml}$. After 7 days, the spheres appeared.

Flow cytometry. Glioma cells were stained with fluorochrome-conjugated antibodies (Abs) for CD133 (Abcam, Cambridge, MA, USA), Nestin (Santa Cruz Biotechnology, Inc., Santa Cruz, CA, USA), Olig2 (Santa Cruz), glial fibrillary acidic protein (GFAP) (Zhongshan Jinqiao Biotech, China), microtubule-associated protein 2 (MAP2) (Millpore, MA, USA) or myelin basic protein (MBP) (Santa Cruz) or control Ab (B\&D, USA). Data were acquired on a Gallios flow cytometer (Beckman Coulter, Miami, FL, USA) and analyzed with FlowJo software.

Immunohistochemisty and immunofluorescence. Glioma cells were incubated with Abs for CD133 (Abcam), Nestin (Santa Cruz), Olig2 (Santa Cruz), GFAP (Zhongshan Jinqiao Biotech), MAP2 (Millpore) or MBP (Santa Cruz). Negative controls were performed by replacing the specific primary Abs with isotype matched control Abs at the same concentration. The primary Abs bound on tumor cells were reacted with FITC or Cy3-conjugated goat anti-rabbit or anti-mouse IgG (Sigma, St. Louis, MO, USA). The cells were then counterstained with 4',6-diamidino-2-phenylindole (DAPI, Sigma) to reveal the nuclei. Immunohistochemistry (IHC) was performed on paraffin-embedded sections. The tumor sections were incubated with Abs for CD3 (Beijing Biosynthesis Biotech, China), CD20 (Santa) or Iba1 (Abcam), followed by detection using a ChemMate Detection kit (Dako, Glostrup, Denmark). Negative controls were performed as previously mentioned. A positive reaction was indicated by brown color using DAB and was counterstained with hematoxylin.

Western blotting. The tumor tissues were resuspended in lysis buffer (Pierce, Rockford, IL, USA). After centrifugation, the supernatants were dissolved in Laemmli sample buffer (Pierce) and heated at $95^{\circ} \mathrm{C}$ for $5 \mathrm{~min}$. Equal amounts of cellular proteins were separated by $10 \%$ SDS-PAGE, immunoblotted with Abs for IL-2 (Beijing Biosynthesis Biotech), IL-4 (Santa Cruz), B7-1 and IL-8 (Santa Cruz) and visualized with a commercial ECL kit (Pierce). To confirm that each lane received the same amount of proteins, the blots were stripped and reprobed with rabbit anti-human/mouse GAPDH Ab (Santa Cruz).

Colony formation assay and characterization of resistance to cytotoxic agents. Cultured cells were made into single cell suspension in defined stem cell medium. Cell density was adjusted into 10 cells $/ \mathrm{ml}$. Seeded $100-\mu 1$ cell suspension in every well of 96-well plate and marked the wells with a single cell. Two weeks later, neurospheres and small colonies were scored as colony-forming unit. Cells (5,000/well) were plated in $100 \mathrm{ml}$ per well in 96-well plates and Nimustine were added. Cell viability was measured after $72 \mathrm{~h}$ with the CellTiter 96 Aqueous Non-radioactive assay (Promega).

Orthotopic glioma implantation. All procedures involving mice were conducted in accordance with the Guidelines of Animal Experiments of Third Military Medical University. Glioma cells were injected orthotopically into the brains of 6-week-old female C57/BL6 mice or female NOD/SCID mice $(\mathrm{n}=5$ each group, Center of Experimental Animals, Third Military Medical University, Chongqing, China). Mice were maintained for 100 days. The brains of euthanized mice were collected and fixed in $4 \%$ paraformaldehyde for the subsequent preparation of sections.

Statistical analysis. Data were analyzed with SPSS10.0 statistical software. When two groups were compared, the unpaired dependent sample $\mathrm{t}$-test was used. $\mathrm{P}<0.05$ was considered statistically significant.

\section{Results}

Stem cell markers were upregulated in GL261-NS and $P G B M-N S$. We seeded GL261 cells and decreased one half of FBS concentration every 3 days. When the FBS concentration was reduced to $2.5 \%$, cell clusters at the top of adhesive cells (Fig. 1A) were collected and suspended in defined stem cell medium. These single cells developed into neurospheres containing 10-20 cells after 6 days (Fig. 1B). After 2 weeks, the size of these spheres expanded 10- to 30 -fold (Fig. 1C). Immunofluorescence staining showed majority of tumor cells in neurospheres were positive for CD133, Nestin and Olig2 (Fig. 1D-F). Flow cytometry confirmed that the percentages of GL261-NS that were positive for GFAP, MAP2 and MBP were, respectively, 2.1 \pm 0.7 , $1.7 \pm 0.6$ and $0.3 \pm 0.07 \%$ (Fig. $1 \mathrm{~J}-\mathrm{L}$ ). However, the percentage of GL261-NS was positive for CD133, Nestin and Olig2 were $25.3 \pm 3.9,72.5 \pm 6.8$ and $81.3 \pm 5.5 \%$, respectively (Fig. 1G-I). When cultured in medium with FBS, GL261-NS adhered to the bottom of culture flask. After 5 days, all the cells in the neurosphere migrated out from the spheres and differentiated into GL261-AC (Fig. 2A and B). Immunofluorescence staining confirmed GL261-AC expressed only slightly stems cell markers (data not shown) and differentiated into cells 
Table I. Summary of specimens examined.

\begin{tabular}{|c|c|c|c|c|c|c|c|}
\hline $\begin{array}{l}\text { Patient } \\
\text { no. }\end{array}$ & Code & $\begin{array}{l}\text { Age (years)/ } \\
\text { gender }\end{array}$ & $\begin{array}{l}\text { Histology/ } \\
\text { WHO grade }\end{array}$ & Location & $\begin{array}{l}\text { CD133-positive } \\
\text { cells in P-NS (\%) }\end{array}$ & $\begin{array}{c}\text { Nestin-positive } \\
\text { cells in P-NS (\%) }\end{array}$ & $\begin{array}{c}\text { Olig2-positive } \\
\text { cells in P-NS }(\%)\end{array}$ \\
\hline 1 & G14 & $52 / \mathrm{F}$ & GBM/IV & Temporal L & 59.30 & 71.60 & 54.90 \\
\hline 2 & G95 & $50 / \mathrm{F}$ & GBM/IV & Frontoparietal R & 21.60 & 59.10 & 70.60 \\
\hline 3 & G74 & $44 / \mathrm{M}$ & GBM/IV & Occipital R & 73.50 & 77.30 & 92.50 \\
\hline 4 & G20 & $72 / \mathrm{M}$ & GBM/IV & Temporal L & 63.30 & 53.30 & 46.70 \\
\hline 5 & G103 & $40 / \mathrm{F}$ & GBM/IV & Frontal L & 17.70 & 82.70 & 12.30 \\
\hline Total & & & & & $47.1 \pm 25.6$ & $68.8 \pm 12.3$ & $55.4 \pm 29.8$ \\
\hline
\end{tabular}

M, male; F, female; WHO, World Health Organization; GBM, glioblastoma; R, right; L, left; P-NS, primary neurosphere.
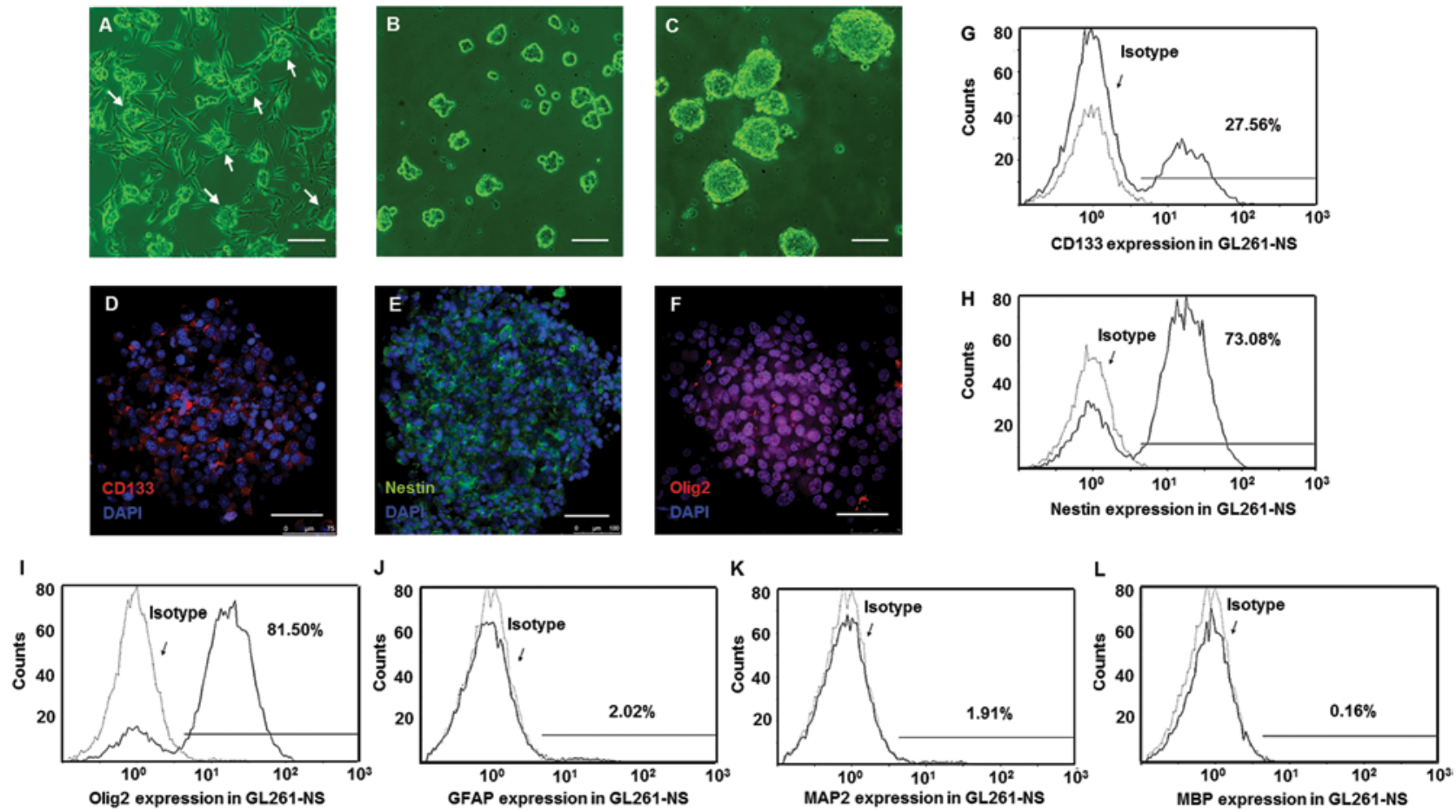

Figure 1. GICs were enriched in GL261-NS and expressed stem cell markers. The clusters (arrows) of GL261 cells appeared in the medium with low concentration of FBS (A), neurospheres (GL261-NS) derived from the clusters and expanded in defined stem cell medium (B and C). Immunofluorescence of stem cell makers CD133 (D, red), Nestin (E, green) and Olig2 (F, red). Flow cytometry analysis showed the percentage of GL261-NS expressing CD133, Nestin and Olig2 (G-I), the percentage of GL261-AC expressing GFAP, MAP2 and MBP (J-L). (A-C) Phase contrast microscopy; scale bar, $200 \mu \mathrm{m}$. (D-F) Merged images, counterstained with DAPI showing nuclei in blue. Laser confocal scanning microscopy; scale bar, $100 \mu \mathrm{m}$.

that were positive for GFAP, MAP2 and MBP (Fig. 2C-E). Flow cytometry showed that the percentage of GL261-AC positive for GFAP, MAP2 and MBP was 76.3 $\pm 6.5,64.6 \pm 7.7$ and $8.3 \pm 1.7 \%$, respectively (Fig. $2 \mathrm{~F}$ ). However, the percentage of GL261-AC positive for CD133, Nestin and Olig2 was $1.3 \pm 0.4,27.1 \pm 5.6$ and $3.9 \pm 0.8 \%$ respectively (Fig. $2 \mathrm{G}$ ). We also isolated PGBM-NS from five GBM multiform patients (Table I). The PGBM-NS expressed CD133 (47.1 $25.6 \%$ ), Nestin $(68.8 \pm 12.3 \%)$ and Olig2 (55.4 $\pm 29.8 \%)$. It is noteworthy that the percentage of PGBM-NS positive for stem cell markers largely varied among individuals (Fig. 2J).

GL261-NS and PGBM-NS exhibit increased self-renewal potential and chemoresistance. The ability of colony forma- tion in define stem cell medium represented the self-renewal potential (13), we seeded GL261-NS and GL261-AC in suspension cultures, GL261-NS showed an 21-fold increase in tumorsphere-forming capacity compared to GL261-AC (64.7 \pm 7.7 versus $3.3 \pm 1.5$ spheres per 100 cells; Fig. $3 \mathrm{~A}$ and B). Although PGBM-NS also possessed a self-renewal potential as strong as GL261-NS (59.0 \pm 13.0 spheres per 100 cells), PGBM-AC showed a higher percentage of colony-forming units $(14.6 \pm 5.2$ spheres per 100 cells) than GL261-AC. So, PGBM-NS showed only an $\sim 4$-fold increase in tumorsphereforming capacity compared to PGBM-AC (Fig. 3B). It has been reported that drug treatment of glioma cell populations induces enrichment of GICs $(8,14)$. We found that GL261-NS and PGBM-NS were both more resistant than GL261-AC and 

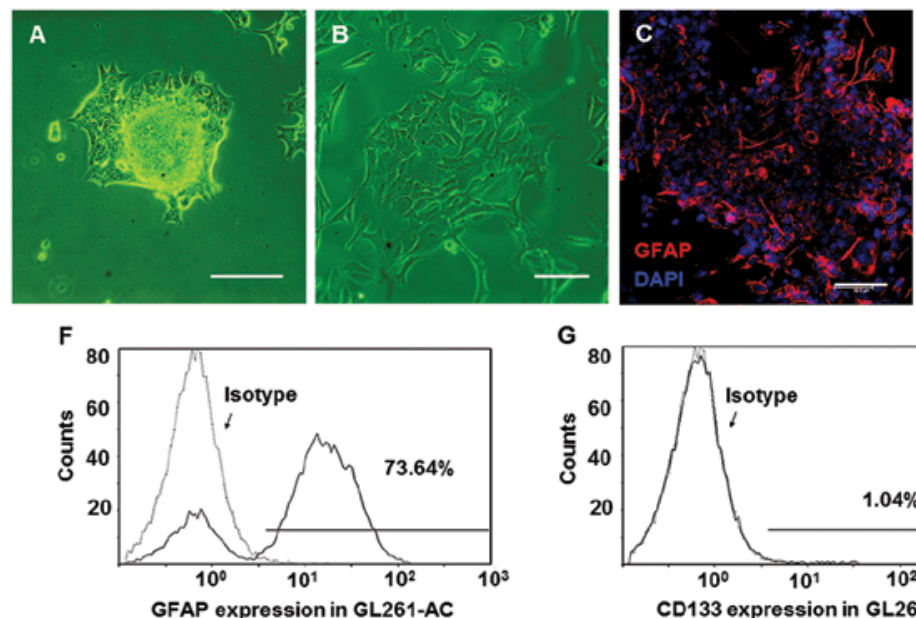

G

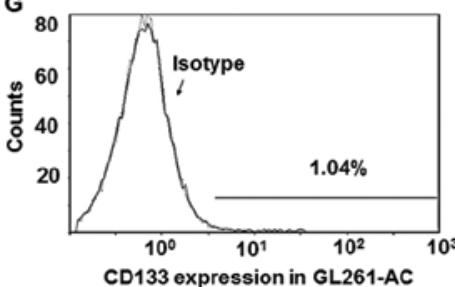

CD133 expression in GL261-AC

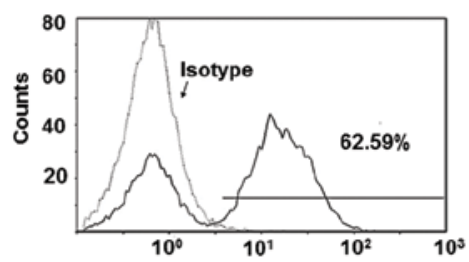

MAP2 expression in GL261-AC

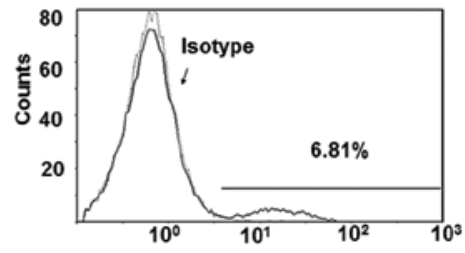

MBP expression in GL261-AC

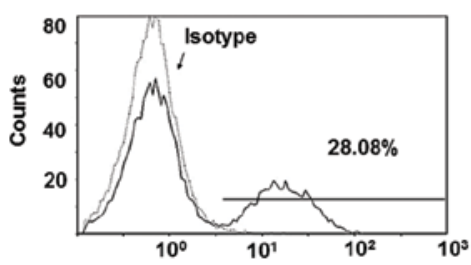

Nestin expression in GL261-AC

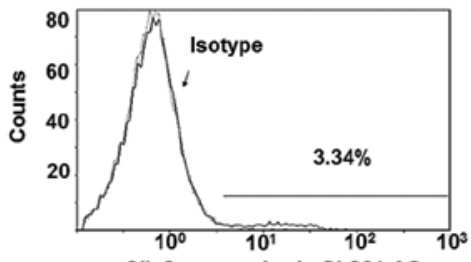

Olig2 expression in GL261-AC
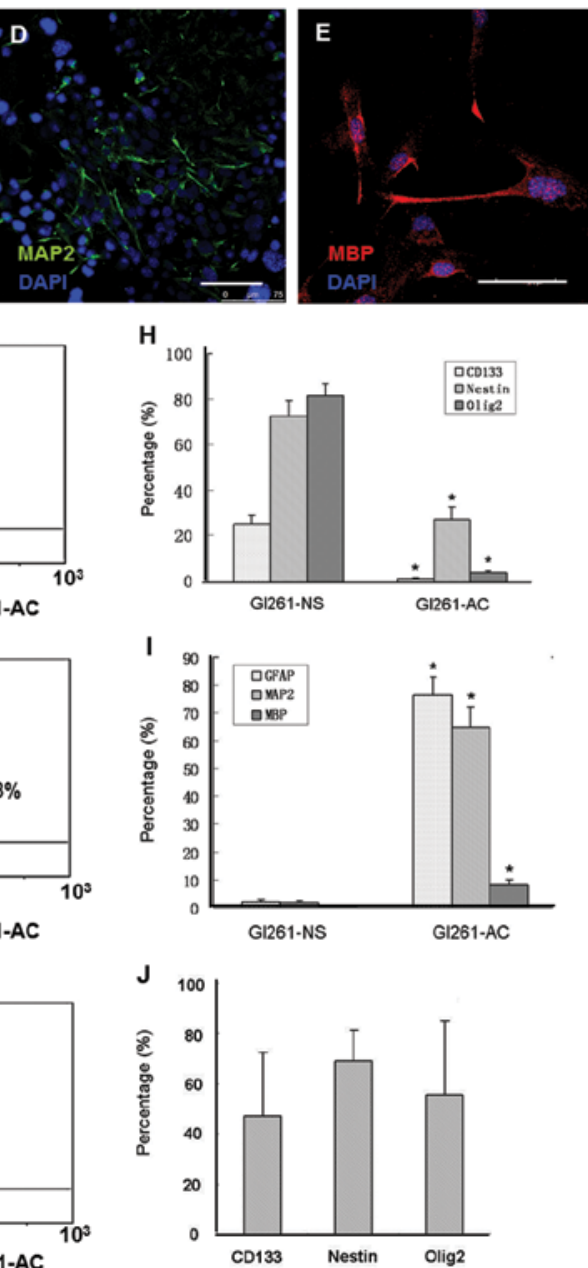

Figure 2. GL261-NS possesses multi-lineage differentiation potential. GL261-AC spilled out from the edges of tumorspheres at $24 \mathrm{~h}$ after plating in DMEM/ F12 containing 10\% FBS (A). The cells in the neurosphere migrated from the spheres and differentiated into GL261-AC in 5 days (B). Immunofluorescence of differentiation makers GFAP (C, red), MAP2 (D, green) and MBP (E, red). Flow cytometry analysis showed the percentage of GL261-AC expressing GFAP, MAP2 and MBP (F and I), the percentage of GL261-AC expressing CD133, Nestin and Olig2 (G and H). The percentage of human primary neurosphere-like glioma cells expressing CD133, Nestin and Olig2 (J). (A and B) Phase contrast microscopy; scale bar, $100 \mu \mathrm{m}$. (C-E) Merged images, counterstained with DAPI showing nuclei in blue. Laser confocal scanning microscopy; scale bar, $75 \mu \mathrm{m}$.

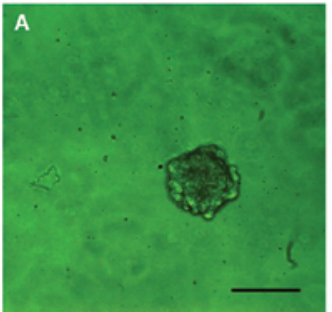

C

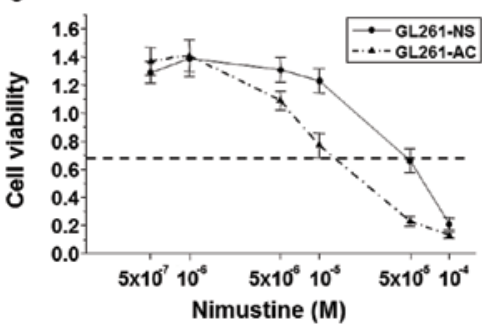

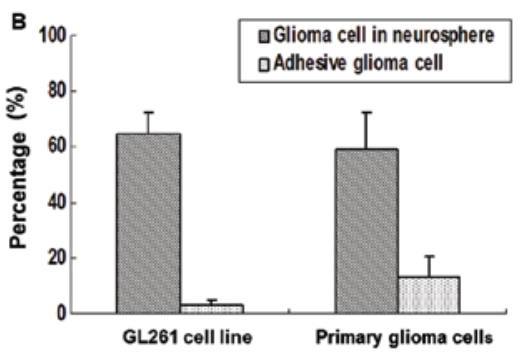

D

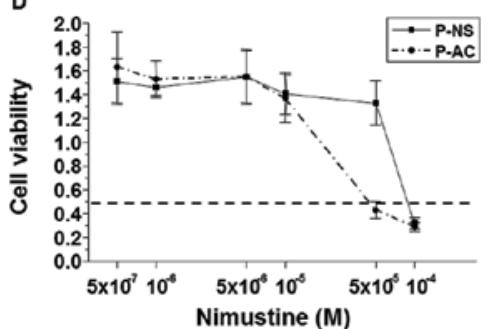

Figure 3. GL261-NS and PGBM-NS show enhanced self-renewal and chemoresistance. Colony formation assay showed GL261-NS could derive from a single cell (A), GL261-NS showed an 21-fold increase in colony-forming capacity compared to GL261-AC and PGBM-NS showed an $~ 4-$ fold increase in colonyforming capacity compared to PGBM-AC (B). * P<0.05 vs. glioma cells in neurosphere by two-tailed t-test. Compared with GL261-AC, GL261-NS showed $\sim 30$-fold increase in $\mathrm{IC}_{50}$ of nimustine (C), PGBM-NS showed $\sim 3$-fold increase in $\mathrm{IC}_{50}$ of nimustine (D). * $\mathrm{P}<0.05$ vs. neurosphere group by two-tailed t-test. (A) Phase contrast microscopy; scale bar, $200 \mu \mathrm{m}$. 
PGBM-AC to nimustine, the commonly used chemotherapeutic drugs. Primary glioma cells possessed stronger resistance to nimustine than GL261 cells. GL261-NS showed 30-fold increase in $\mathrm{IC}_{50}$ of nimustine compared with GL261-AC, whereas PGBM-NS showed only 3 -fold increase in $\mathrm{IC}_{50}$ of nimustine compared with PGBM-AC (Fig. 3C and D).

Neurosphere-like glioma cells show more enhanced tumorigenicity in syngeneic graft model than in heterogeneous immunodeficiency model. Many studies have reported that PGBM-NS showed enhanced tumorigenicity in immunodeficient mice (15-18). We postulated that this model might not be able to reveal the biological feature of GICs accurately because of immunodeficiency and individual differences among patients. To test this hypothesis, we assessed the functional potential of GICs by detecting their in vivo tumorseeding ability. We confirmed that GL261-NS possessed stronger tumorigenicity than GL261-AC in C57/BL6 mice. Hemorrhage and necrosis appeared at the inoculation sites in the $5 \times 10^{3}$ GL261-NS injection group. However, there is no abnormality in the inoculation sites of GL261-AC injection group (Fig. 4A). The xenografts derived from GL261-NS showed the typical features of human GBM, obvious cellular atypia, high density of micro-vessels and numerous abnormal mitotic figures. The margins between tumor and normal brain tissue were unclear, showing intraparenchymal invasion pattern of the tumors. In contrast, tumors formed by GL261-AC rarely showed histological characteristics of malignant glioma (Fig. 4A). Kaplan-Meier survival analysis confirmed that GL261-NS is significantly more aggressive than GL261-AC in C57/BL6 mice. The median survival of mice injected with $5 \times 10^{3}$ GL261-NS was $48.8 \pm 11.8$ days, whereas only one mouse injected with $5 \times 10^{3}$ GL261-AC was dead at 56 days after inoculation, the other four mice were all alive at 100 days $(\mathrm{P}<0.01)$. However, we did not find significant difference in survival time between $5 \times 10^{4}$ GL261-NS group (23.4 \pm 4.6 days) and 5x10 4 GL261-AC group (29.8 \pm 6.4 days, $\mathrm{P}=0.105$ ) (Fig. 4B). Unexpectedly, GL261-NS did not show enhanced tumorigenicity in immunodeficient mice, there was no significant difference in survival time of NOD/SCID mice between $5 \times 10^{3}$ GL261-NS group (35.4 \pm 5.4 days) and $5 \times 10^{3}$ GL261-AC group (37.8 \pm 5.5 days, $\left.\mathrm{P}=0.505\right)$, the survival time of NOD/SCID mice was shorter than C57/BL6 mice when injected with the equal number of tumor cells (Fig. 4C). We inoculated orthotopically $3 \times 10^{4}$ GL261-AC and $3 \times 10^{4}$ GL261-NS respectively into C57/BL6 mice, and treated these mice with nimustine $(5 \mathrm{mg} / \mathrm{kg})$ or vehicle after 7 days of inoculation with daily administration. Nimustine-treated animals seeded with GL261-AC showed an obvious trend toward better prognosis compared to vehicle-treated animals, the survival time was from $29.8 \pm 6.4$ to $43.8 \pm 9.1$ days. But the treatment of nimustine had less impact on survival in animals injected with GL261-NS, which indicated GL261-NS possessed increased drug resistance relative to GL261-AC in vivo (Fig. 4D). Tumorseeding assay with different cellular amounts indicated that tumors could be generated with $1 \times 10^{2}$ GL261-NS in C57/ BL6 mice, which was 50-fold less than the cellular number of GL261-AC that was required for tumor survival. However, the discrepancy of tumor-seeding ability between GL261-NS and GL261-AC did not appear in NOD/SCID mice. The amount of
PGBM-NS that was needed to generate a tumor was $\sim 10$-fold less than that of PGBM-AC in NOD/SCID mice (Fig. 4E).

Glioma derived from GL261-NS in C57/BL6 mimicked the primary human glioma histologically better than glioma derived from PGBM-NS in NOD/SCID mice. Grafts formed by GL261-NS and PGBM-NS both grew rapidly with pleomorphism and high density of microvessels, which are typically seen in human GBM multiforme (Fig. 5A-C). Immunocytes and immunomediators that are indispensable components of the neoplastic microenvironment can modulate the biological behavior of malignances (19-21). IHC showed various immunocytes, including macrophage, DC, B cell and T cell, infiltrated into the tumor region of primary GBM (22-24). Similar composition and percentage of infiltrating immunocytes could be detected in grafts formed by GL261-NS in C57/ BL6 mice, but not in grafts formed by human PGBM-NS in immunodeficient mice (Fig. 5A-C). Similarly, many kinds of immunomediators, such as IL-2, IL-4, B7-1, IL-8, which play important roles in human primary glioma immunity were absent or reduced in grafts formed by human PGBM-NS in immunodeficient mice (Fig. 5D and E). Because of the lack of impacts from immune system, the orthotopic implantation of human PGBM-NS in immunodeficient mice may not be a reliable model to evaluate the biological characteristics of GICs in vivo, the syngeneic glioma implantation model made by GL261-NS in C57/BL6 mice was more desirable.

\section{Discussion}

The high incidence, high mortality and limited advances in glioma treatment impelled us to inspect the methodology and technology of glioma research (25). GICs have been recognized as the insurmountable obstacle for glioma curability (26-28). The experience from the normal stem cell research indicates the stem cell niche is essential for regulating the biological behavior of stem cell, the stem cells which break away from the niche can not maintain stemness and original characteristics (29). Recent studies confirmed inflammatory cells are the indispensable components of the tumor stem cell niche, inflammatory cells and inflammatory mediators regulate the malignant behavior of tumor stem cells $(30,31)$. However, the conventional model for in vivo assay is engrafting GICs into immunodeficient animals. It is unclear whether the research model lacking regulation of immune system can reflect the malignant behavior of GICs in vivo. We found that GICs were enriched in GL261-NS which showed potential for self-renewal and multi-directional differentiation. GL261-NS expressed neural stem cell markers and possessed stronger chemoresistance than GL261-AC in vitro and in vivo. The differences of tumor-seeding ability between GICs and adhesive glioma cells were shown more adequately in the syngeneic graft model, compared with the heterogenic immunodeficient model. Inflammatory components of glioma in C57/BL6 mice mimicked primary GBM much more closely than the components in NOD/SCID mice.

Animal models of glioma can be divided into three categories: the induced spontaneous tumor model, the engrafted tumor model and the genetically engineered tumor model (4). The spontaneous glioma model can be induced by chemical 

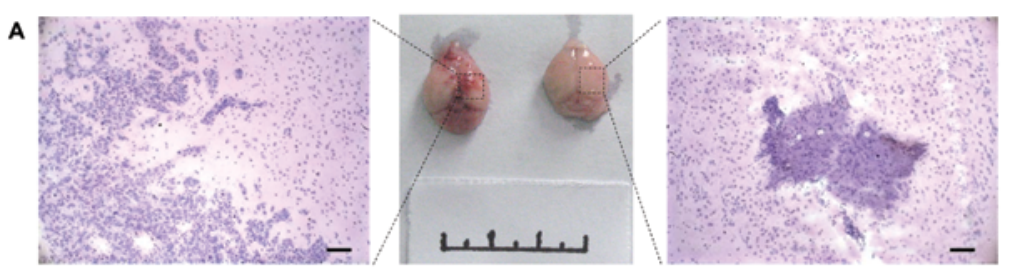

B 50,000 GL261-AC in C57/BL6 mice $\begin{aligned} & 50,000 \mathrm{GL} 261-\mathrm{NS} \text { in C57/8L6 mico } \\ & 5,000 \mathrm{GL} 261-\mathrm{AC} \text { in C } 57 / 8 \mathrm{BL} 6 \text { mice }\end{aligned}$ $\mathrm{C}+5,000 \mathrm{GL} 261-\mathrm{AC}$ in NOD-SCID mico 5,000 GL261-AC in C57/8L6 mice - 5,000 GL261-NS in NOD-SCID mico

D GL261-NS in control group

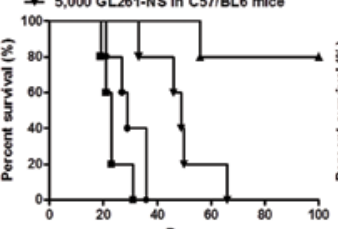
5,000 GL261-NS in C57/8L6 mice
5,000 GL261-AC in C57/8L6 mice
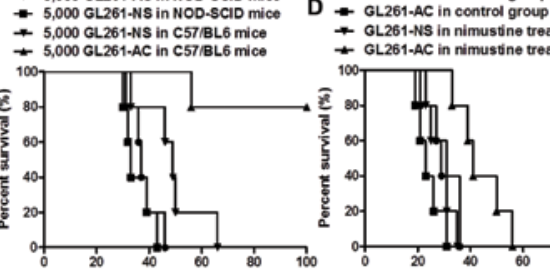

- GL261-NS in nimustine treated group

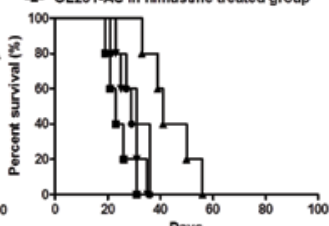

$\mathbf{E}$

\begin{tabular}{|c|c|c|c|c|c|c|}
\hline & \multicolumn{2}{|c|}{ C57/BL6 mice } & \multicolumn{2}{c|}{ NOD-SCID mice } & \multicolumn{2}{c|}{ NOD-SCID mice } \\
\hline Cell injected & GL261-NS & GL261-AC & GL261-NS & GL261-AC & PGBM-NS & PGBM-AC \\
\hline $5 \times 10^{4}$ & $5 / 5$ & $5 / 5$ & $5 / 5$ & $5 / 5$ & $5 / 5$ & $5 / 5$ \\
\hline $1 \times 10^{4}$ & $4 / 4$ & $4 / 4$ & $5 / 5$ & $5 / 5$ & $5 / 5$ & $5 / 5$ \\
\hline $5 \times 10^{3}$ & $5 / 5$ & $1 / 5$ & $5 / 5$ & $5 / 5$ & $4 / 5$ & $2 / 5$ \\
\hline $1 \times 10^{3}$ & $4 / 5$ & $0 / 5$ & $5 / 5$ & $3 / 5$ & $3 / 5$ & $0 / 5$ \\
\hline $5 \times 10^{2}$ & $3 / 5$ & $0 / 5$ & $2 / 5$ & $1 / 5$ & $1 / 5$ & $0 / 5$ \\
\hline $1 \times 10^{2}$ & $1 / 5$ & $0 / 5$ & $2 / 5$ & $0 / 5$ & $0 / 5$ & $0 / 5$ \\
\hline
\end{tabular}

Figure 4. The tumorigenicity of GICs in the syngeneic immunocompetent mice and the immunodeficient mice. (A) GL261-NS showed stronger tumorigenicity than GL261-AC in C57/BL6 mice. The typical features of human GBM could be found in tumor derived from GL261-NS. Kaplan-Meier survival curves of C57/BL6 mice with the different amounts of GL261-NS and GL261-AC implantation, P<0.01 (B). Kaplan-Meier survival curves of C57/BL6 and NOD/SCID mice with the same amount of GL261-NS and GL261-AC injection, P<0.01 (C). Kaplan-Meier survival curves of GL261-NS and GL261-AC tumor-bearing C57/BL6 mice with nimustine or control treatment, $\mathrm{P}<0.01$ (D). Tumor-seeding assays with different cellular amounts in C57/BL6 mice and NOD/SCID mice (E). (A) Hematoxylin counterstain; scale bar, $100 \mu \mathrm{m}$.

A
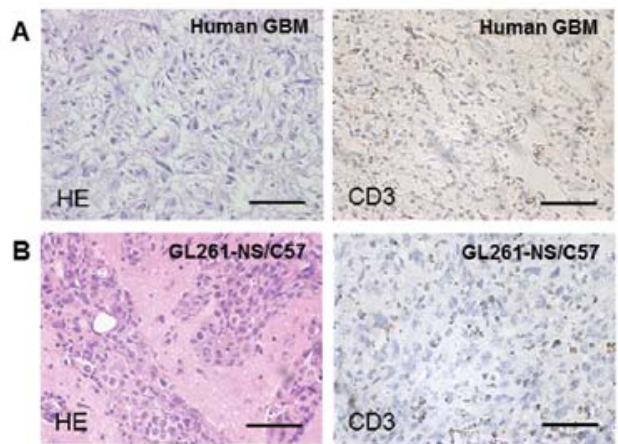

\section{C}
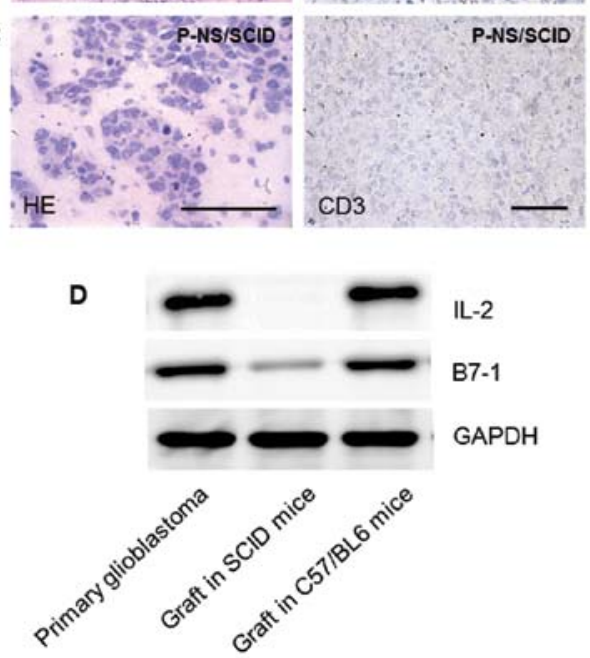
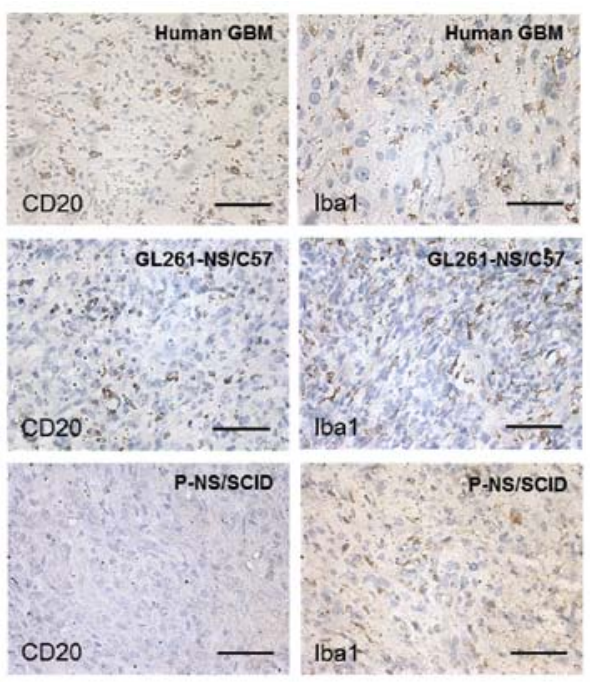

$\mathbf{E}$
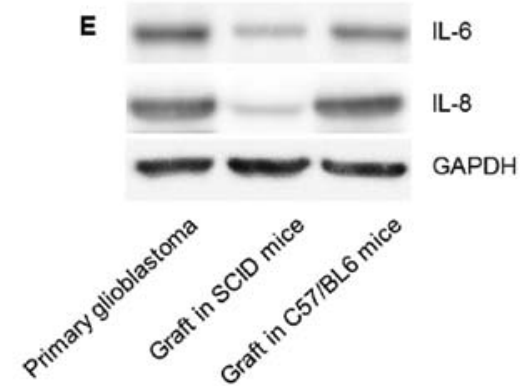

Figure 5. Glioma derived from GICs in syngeneic model mimicked better the human GBM histologically. CD3, CD21 and Iba1 immunoreactivity was observed in human GBM (A) and glioma derived from GL261-NS in syngeneic model (B), but CD3 and CD21 was rarely detected in xenografts derived from PGBM-NS in immunodeficient model (C-E). Western blotting showed the expression of IL-2, IL-6, IL-8 and B7-1 in human GBM, GL261-NS tumor in syngeneic model and PGBM-NS tumor in immunodeficient model. (A-C) Hematoxylin counterstain; scale bar, $100 \mu \mathrm{m}$. 
reagents or oncogenic virus, the course of progress and histological phenotype of spontaneous glioma model are similar to primary human glioma. However, the ambiguous genetic background and prolonged incubation limit its application. The genetically engineered tumor model is created by transgenic technology allowing the investigator to activate or turn off certain specific genes and produce the animal model with specific genetic pattern of primary human glioma $(32,33)$. However, there are still great differences in pathogenesis between the genetically engineered model and human tumor (4). Based on the good repeatability and the high penetrance, the engrafted model is still the most wildly used model for evaluating new therapeutic concepts for glioma (34). The engrafted models are the xenograft model and the syngraft model. The failure of existing therapies necessitates investigation of new therapeutic strategies for glioma. Among these promising strategies are the activation of the specific immune response to GICs or reversing the immunosuppressive GIC-niche $(35,36)$. However, the first challenge is that animal models of primary GICs faithfully reflect the interaction between GICs and the host's immune system. The pan-immune deficiency in the NOD/SCID or athymic mice obviously restricts the application of the xenograft models. A more reliable animal model for research on GICs is needed.

The GL261 originates from the brain of C57/BL6 mice, and is cultured by serial syngeneic transplantation of tumor pieces (37). The genetic mutation pattern of GL261 is clear. GL261 is a moderate immunogenic tumor cell line with low level of MHC class I molecules, absent MHC class II molecules and moderate level of costimulatory molecules (38). Approximately 30\% of GL261 cells expressed the neural stem cell marker Nestin and could be cultured into the suspension neurosphere-like cells (GL261-NS) in stem cell defined medium. Similar to human primary glioma cells, GL261-NS has a relatively high fraction of $\mathrm{CD} 133^{+}$glioma cells, which are candidate GICs. This cell population has been reported to be low immunogenic, thus the tumor derived from GICs may model the human condition and recapitulate the immune status of glioma more reliably (39). Tumor immunoediting plays crucial roles in the process of tumorigenesis and progression (40). The immune pressure eliminates nascent tumor cells and results in tumor progression with reduced immunogenicity. Immune system not only eradicates tumor cells, but also shapes the malignant disease and screens out the subclone glioma cell that can gain growth advantage. The xenografts, which derived by implanting PGBM-NS into immunodeficient animal, may ignore the immune shaping process and can not mimic the progression of primary human glioma objectively. The innate and specific immune responses in the syngeneic model make this model an improvement over xenograft models to reflect the interaction between GICs and immune system and to investigate promising immunotherapy on GICs. Our model confirmed that the incubation period of GL261-NS glioma in C57/BL6 mice is longer than that in NOD/SCID mice (data not shown). GL261-AC could survive and form a tumor in immunodeficient mice, but the same amount of GL261-AC could not grow into a tumor in immunocompetent mice. The prognosis of immunodeficient mice bearing glioma derived by GL261-NS was much worse than that of immunocompetent mice. These results suggested that the immune system carried out a selective pressure on GICs in the process of tumorigenesis and progression.

In conclusion, implantation of GL261-NS into C57/BL6 mice is a reliable syngeneic graft model since it is indeed a highly reproducible and easy-to-establish model system that can reflect the interplay of GICs and immune components. However, we should still pay attention to the immunologic changes induced by the implantation itself. The discrepancies in neoplastic mechanism and progression between mouse implantation model and human GBM always exist. Moreover, human gliomas could have different genetic mutation patterns that induce various malignant biologic behavior, we do not imagine modeling the diverse primary gliomas with single one kind of animal model. The above data urge us to modify our animal glioma model further and create different kinds of animal models with specific genetic mutations in order to mimic the human malignant glioma more accurately.

\section{Acknowledgements}

This study was supported by the National Natural Science Foundations of China (NSFC, nos. 30901538 and 81270039) and the medical research from Chongqing Municipal Health Bureau (no. 2011-2-427).

\section{References}

1. Stupp R, Hegi ME, Mason WP, et al: Effects of radiotherapy with concomitant and adjuvant temozolomide versus radiotherapy alone on survival in glioblastoma in a randomised phase III study: 5-year analysis of the EORTC-NCIC trial. Lancet Oncol 10: 459-466, 2009.

2. Stiles CD and Rowitch DH: Glioma stem cells: a midterm exam. Neuron 58: 832-846, 2008.

3. Singh A and Settleman J: EMT, cancer stem cells and drug resistance: an emerging axis of evil in the war on cancer. Oncogene 29: 4741-4751, 2010.

4. Sughrue ME, Yang I, Kane AJ, et al: Immunological considerations of modern animal models of malignant primary brain tumors. J Transl Med 7: 84, 2009.

5. Wei J, Barr J, Kong LY, et al: Glioblastoma cancer-initiating cells inhibit T-cell proliferation and effector responses by the signal transducers and activators of transcription 3 pathway. Mol Cancer Ther 9: 67-78, 2010.

6. Dziurzynski K, Wei J, Qiao W, et al: Glioma-associated cytomegalovirus mediates subversion of the monocyte lineage to a tumor propagating phenotype. Clin Cancer Res 17: 4642-4649, 2011.

7. Pellegatta S, Poliani PL, Corno D, et al: Neurospheres enriched in cancer stem-like cells are highly effective in eliciting a dendritic cell-mediated immune response against malignant gliomas. Cancer Res 66: 10247-10252, 2006.

8. Yu SC, Ping YF, Yi L, et al: Isolation and characterization of cancer stem cells from a human glioblastoma cell line U87. Cancer Lett 265: 124-134, 2008.

9. Bao S, Wu Q, Sathornsumetee S, et al: Stem cell-like glioma cells promote tumor angiogenesis through vascular endothelial growth factor. Cancer Res 66: 7843-7848, 2006.

10. Maes W and Van Gool SW: Experimental immunotherapy for malignant glioma: lessons from two decades of research in the GL261 model. Cancer Immunol Immunother 60: 153-160, 2011.

11. Yi L, Xiao H, Xu M, et al: Glioma-initiating cells: a predominant role in microglia/macrophages tropism to glioma. J Neuroimmunol 232: 75-82, 2011.

12. Yi L, Zhou ZH, Ping YF, et al: Isolation and characterization of stem cell-like precursor cells from primary human anaplastic oligoastrocytoma. Mod Pathol 20: 1061-1068, 2007.

13. Yang S, Wang B, Guan C, et al: Foxp $3^{+} \mathrm{IL}-17^{+} \mathrm{T}$ cells promote development of cancer-initiating cells in colorectal cancer. J Leukoc Biol 89: 85-91, 2011. 
14. Beier D, Schulz JB and Beier CP: Chemoresistance of glioblastoma cancer stem cells - much more complex than expected. Mol Cancer 10: 128, 2011.

15. Galli R, Binda E, Orfanelli U, et al: Isolation and characterization of tumorigenic, stem-like neural precursors from human glioblastoma. Cancer Res 64: 7011-7021, 2004.

16. Calabrese C, Poppleton H, Kocak M, et al: A perivascular niche for brain tumor stem cells. Cancer Cell 11: 69-82, 2007.

17. Krishnamurthy S, Dong Z, Vodopyanov D, et al: Endothelial cell-initiated signaling promotes the survival and self-renewal of cancer stem cells. Cancer Res 70: 9969-9978, 2010.

18. Li Z, Bao S, Wu Q, et al: Hypoxia-inducible factors regulate tumorigenic capacity of glioma stem cells. Cancer Cell 15: 501-513, 2009.

19. Grivennikov SI, Greten FR and Karin M: Immunity, inflammation, and cancer. Cell 140: 883-899, 2010.

20. Bayne LJ, Beatty GL, Jhala N, et al: Tumor-derived granulocytemacrophage colony-stimulating factor regulates myeloid inflammation and $\mathrm{T}$ cell immunity in pancreatic cancer. Cancer Cell 21: 822-835, 2012.

21. Charles NA, Holland EC, Gilbertson R, Glass R and Kettenmann H: The brain tumor microenvironment. Glia 59: 1169-1180, 2011.

22. Hussain SF, Yang D, Suki D, Aldape K, Grimm E and Heimberger AB: The role of human glioma-infiltrating microglia/ macrophages in mediating antitumor immune responses. Neuro-oncol 8: 261-279, 2006.

23. Wiencke JK, Accomando WP, Zheng S, et al: Epigenetic biomarkers of T-cells in human glioma. Epigenetics 7: 1391-1402, 2012.

24. Hong TM, Teng LJ, Shun CT, Peng MC and Tsai JC: Induced interleukin-8 expression in gliomas by tumor-associated macrophages. J Neurooncol 93: 289-301, 2009.

25. Guryanova OA, Wu QL, Cheng L, et al: Non-receptor tyrosine kinase BMX maintains self-renewal and tumorigenic potential of glioblastoma stem cells by activating STAT3. Cancer Cell 19: 498-511, 2011.

26. Lamszus $\mathrm{K}$ and Gunther HS: Glioma stem cells as a target for treatment. Target Oncol 5: 211-215, 2010.

27. Chen J, McKay RM and Parada LF: Malignant glioma: lessons from genomics, mouse models, and stem cells. Cell 149: 36-47, 2012.
28. Li Z, Wang H, Eyler CE, Hjelmeland AB and Rich JN: Turning cancer stem cells inside out: an exploration of glioma stem cell signaling pathways. J Biol Chem 284: 16705-16709, 2009.

29. Wagers AJ: The stem cell niche in regenerative medicine. Cell Stem Cell 10: 362-369, 2012.

30. Ginestier C, Liu S, Diebel ME, et al: CXCR1 blockade selectively targets human breast cancer stem cells in vitro and in xenografts. J Clin Invest 120: 485-497, 2010.

31. Sansone P, Storci G, Tavolari S, et al: IL-6 triggers malignant features in mammospheres from human ductal breast carcinoma and normal mammary gland. J Clin Invest 117: 3988-4002, 2007.

32. Weiss WA, Burns MJ, Hackett C, et al: Genetic determinants of malignancy in a mouse model for oligodendroglioma. Cancer Res 63: 1589-1595, 2003.

33. Hu X, Pandolfi PP, Li Y, Koutcher JA, Rosenblum M and Holland EC: mTOR promotes survival and astrocytic characteristics induced by Pten/AKT signaling in glioblastoma. Neoplasia 7: 356-368, 2005

34. Szatmari T, Lumniczky K, Desaknai S, et al: Detailed characterization of the mouse glioma 261 tumor model for experimental glioblastoma therapy. Cancer Sci 97: 546-553, 2006.

35. Hatiboglu MA, Wei J, Wu AS and Heimberger AB: Immune therapeutic targeting of glioma cancer stem cells. Target Oncol 5: 217-227, 2010 .

36. Wei J, Barr J, Kong LY, et al: Glioma-associated cancer-initiating cells induce immunosuppression. Clin Cancer Res 16: 461-473, 2010.

37. Ausman JI, Shapiro WR and Rall DP: Studies on the chemotherapy of experimental brain tumors: development of an experimental model. Cancer Res 30: 2394-2400, 1970.

38. Daga A, Orengo AM, Gangemi RM, et al: Glioma immunotherapy by IL-21 gene-modified cells or by recombinant IL-21 involves antibody responses. Int J Cancer 121: 1756-1763, 2007.

39. Abdouh M, Facchino S, Chatoo W, Balasingam V, Ferreira J and Bernier G: BMI1 sustains human glioblastoma multiforme stem cell renewal. J Neurosci 29: 8884-8896, 2009.

40. Kim R, Emi M and Tanabe K: Cancer immunoediting from immune surveillance to immune escape. Immunology 121: 1-14, 2007. 